
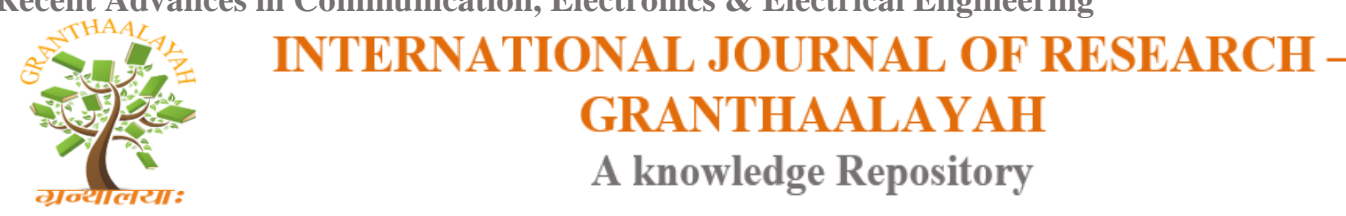

RACEEE - 17

\title{
SOLID STATE RELAY SWITCHES CONTROL USING MSP 430 FOR INSTANTANEOUS HIGH CURRENT SUPPLY
}

\author{
Smitha T.K ${ }^{1}$, Dr. Pritum Shah ${ }^{2}$ \\ Bangalore, India
}

DOI: https://doi.org/10.29121/granthaalayah.v5.i4RACEEE.2017.3335

\begin{abstract}
Microcontroller MSP430 is used to control the SSR switches to supply a very high current. PC is interfaced to the supercomputer for computations. SSR and contactor switches are used to pass the current. Capacitor discharge system is also used to supply a very high current around 170200A. High current is passed to the sample specimen placed inside the heater which needs to be heated at a very high temperature. The current pulse is generated by the capacitor discharge system. Power supply for regular heater is suspended temporarily and capacitor is allowed to discharge using the Capacitor discharge heating. This is achieved by the instructions through the computer to the microcontroller. Current injection at the particular instant is done with the help of a software by initiating an interrupt from the computer. The interrupt pin of the microcontroller becomes high by this and the operation is initiated correspondingly. Thus the MSP430 receives the command to give the necessary pulse of current for heating at the required time. Feedback is received with regard to the position of the switches whether the particular SSR is ON or OFF. Injection of power depends on the corresponding switch positions by giving the necessary instructions by the computer.
\end{abstract}

Keywords: Microcontroller MSP 430; RP; SSR.

Cite This Article: Smitha T.K, and Dr. Pritum Shah. (2017). "SOLID STATE RELAY SWITCHES CONTROL USING MSP 430 FOR INSTANTANEOUS HIGH CURRENT SUPPLY." International Journal of Research - Granthaalayah, 5(4) RACEEE, 130-140. https://doi.org/10.29121/granthaalayah.v5.i4RACEEE.2017.3335.

\section{Introduction}

Microcontroller MSP430, a mixed-signal microcontroller family from Texas Instruments is used to control the solid state relays and contactor switches automatically. MSP 430 is built around a 16-bit CPU and is designed for low cost and, specifically, low power consumption embedded applications. 
Recent Advances in Communication, Electronics \& Electrical Engineering

A computer at the frontline of contemporary processing capacity, a supercomputer is used in which particularly speed of calculation can happen at speeds of nanoseconds.

Systems with massive number of processors generally take one of two paths: In one approach (e.g., in distributed computing), a large number of discrete computers (e.g., laptops) distributed across a network (e.g., the Internet) devote some or all of their time to solving a common problem; each individual computer (client) receives and completes many small tasks, reporting the results to a central server which integrates the task results from all the clients into the overall solution.

\section{Microcontroller MSP 430}

MSP430 is used for low powered embedded devices. Electric current drawn in idle mode is less than $1 \mu \mathrm{A}$. Top CPU speed is $25 \mathrm{MHz}$. It can be throttled back for lower power consumption. MSP430 uses six different low-power modes, which can disable unneeded clocks and CPU. Also, MSP430 is capable of wake-up times below 1 microsecond, allowing the microcontroller to stay in sleep mode longer, minimizing its average current consumption. It is useful in time delay processes [10].

Device comes in a variety of configurations featuring the usual peripherals: internal oscillator, timer including PWM, watchdog, USART, SPI, I²C, 16-bit ADCs, and brownout reset circuitry. Some peripheral options include comparators (that can be used with the timers to do simple ADC), on-chip op-amps for signal conditioning, 12-bit DAC, LCD driver, hardware multiplier, USB, and DMA for ADC results.

In the proposed work, the microcontroller MSP 430 plays a very important role in controlling the switches and getting the feedback.

Also MSP 430 monitors the position of the switches and decides to operate the particular switch at the particular instant of time. By this the inrush of current can be avoided and the necessary amount current can be supplied without damaging the switches.

Fig 1 shows the functional block diagram of the microcontroller MSP 430.

It shows the different IO ports, ADC, RAM, Flash memory, Clock system, Timers, Brown-out protection, Comparator, CPU, JTAG interface, Emulation, Spy-Bi-Wire etc. 
Recent Advances in Communication, Electronics \& Electrical Engineering

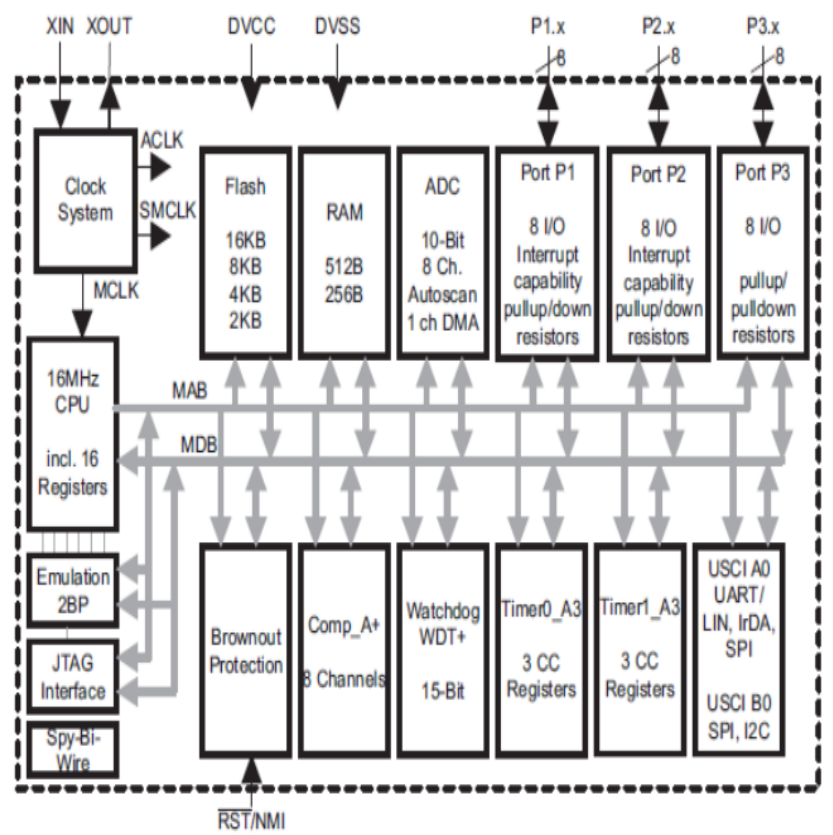

Figure 1: Functional Block Diagram of MSP 430

\subsection{Microcontroller Based Switching}

Microcontroller MSP 430 is used to control the switches. A control program is written to control 2 SSR switches. A feedback control is given to check the position of the SSR switches. The program also decides whether to inject power or not by the corresponding switch positions. Instructions for all these are given by the computer.

In future Remote control system could be adopted for MSP430 which makes the work simpler [11].

\section{Supercomputer}

Scientific Computing deals with solving complex scientific problems which can take months to create and debug. Supercomputers play an essential role in high-performance computing. In general, modern supercomputers are built as a cluster system, which is a system of multiple computers interconnected on a network. In coding a parallel program [13] on such a cluster system, MPI (Message Passing Interface) is utilized.

Processing capacity in a supercomputer can happen at trillions of floating point operations per second.

Supercomputers play an important role in the field of computational science, and are used in various fields, including quantum mechanics, weather forecasting, oil and gas exploration, molecular modelling and physical simulations. Computations can be done in fraction of seconds [15]. 
Recent Advances in Communication, Electronics \& Electrical Engineering

A protocol that one program can use to request a service by the use of a program that is placed in alternative computer in a network without the need to know network details is the Remote Procedure Call (RPC). A procedure call is also sometimes known as a subroutine call or a function call. The client/server model is used by the RPC. Client is the requesting program and the server is the service-providing program. An RPC is a synchronous operation that requires the requesting program to be held till the remote procedure results are returned, like a regular or local procedure call. However, multiple RPCs can be performed concurrently by the use of lightweight processes or threads that share the same address space.

The compiled code includes a stub which serves as the remote procedure code's representative when the program statements which utilize RPC are compiled to an executable program. The stub accepts the request and sends it to a client runtime program in the local computer once the program is made to run and the procedure call is given. The runtime program of the client has the information of addressing the remote computer as well as server application and delivers the message through the network that requests the remote procedure. Likewise, the stub is included in the server which interfaces with the remote procedure and a runtime program. Results are returned in the same way.

Supercomputer provides an infrastructure for sharing tremendous amounts of resources (e.g., computing, storage, and data) distributed over the Internet. Efficiency, Fidelity, and Flexibility with high performance can be achieved by the use of supercomputer.

In this work the data obtained from the experiment by the computer is sent to the supercomputer so that it does the necessary calculations in a very short span of time in the order of nanoseconds and returns the result to the computer where in different parameters can be recorded and plotted. Computer acts like a client and the supercomputer acts as a server. This works using remote procedure call (RPC) [14].

\section{Solid State Relay}

An electronic switching device, a solid state relay (SSR) switches on or off when a small external voltage is applied across its control terminals. SSRs consist of a sensor which responds to an appropriate input (control signal), a solid-state electronic switching device which switches power to the load circuitry, and a coupling mechanism to enable the control signal to activate this switch without mechanical parts. The relay is designed to switch either AC or DC to the load. It performs the same function as an electromechanical relay, but has no moving parts. Solid state relay is used to switch into various numbers of switched capacitor cells and power MOSFET switches [12].

SSR's operate very fast with no sparking and increased lifetime. SSR's are inherently small and has constant output resistance.

There are different types of SSR's, among which photo-coupled or optically coupled SSR's are used here. Advantage of using optically coupled SSR is that sensitivity ranges from about 6milliwatts (e.g., 3 volts dc at $2 \mathrm{~mA}$ ) to 100 milliwatts. It is also compatible with TTL logic levels and can be driven in parallel by high-fan-out logic lines. 
Recent Advances in Communication, Electronics \& Electrical Engineering

Here one end of the SSR is interfaced with the microcontroller MSP 430 and the other end is connected to the contactor.

\section{Contactor}

A contactor similar to a relay is an electrically controlled switch used for switching a power circuit, except with higher current ratings. A contactor is controlled by a circuit that has much lower power level than the switched circuit. Contactor is mainly used for arc suppression in case of high current flow [6]. Contactor used for arc-erosion experiments in [16] can operate up to 40000 operations.

Contactors range from those having a breaking current of several amperes to thousands of amperes and $24 \mathrm{~V}$ DC to many kilovolts. Because arcing and consequent damage occurs just as the contacts are opening or closing, contactors are designed to open and close very rapidly; there is often an internal tripping point mechanism to ensure rapid action.

\section{Design and Implementation}

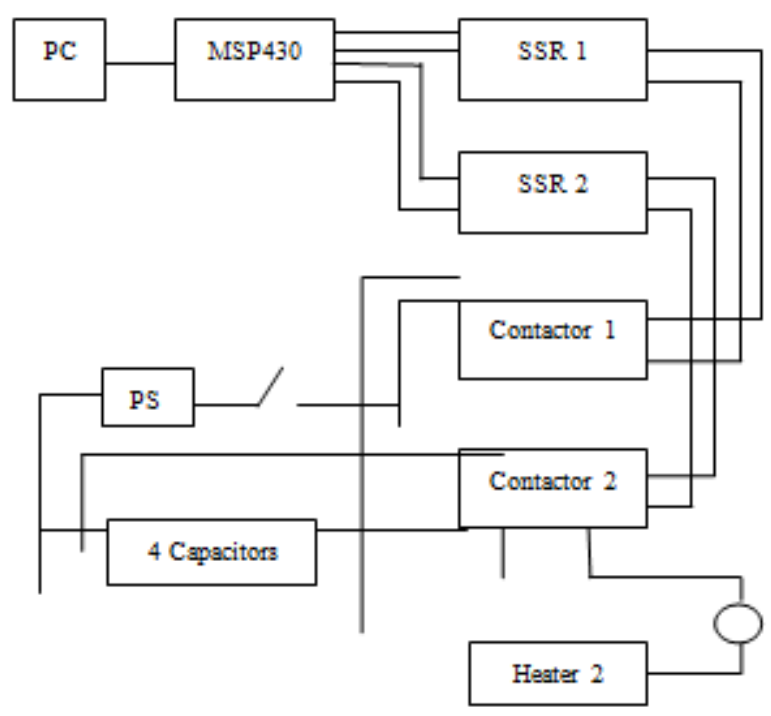

Figure 2: Main Block Diagram of the Design

Microcontroller MSP 430 plays an important role here in controlling the solid state relay switches and contactors. A very high current around $170 \mathrm{~A}$ is required to be supplied to the sample specimen (Methane Hydrate) that is placed inside the pyrophyllite in the cylinder. Specific heat of the sample is more important [7] [8]. Multiple switches are used in parallel combination in order to supply a very high current. Solid state relays and contactors are used as switches. As shown in Fig 2 microcontroller MSP 430 is interfaced to computer on one end [5], and the other end is connected to the solid state relay switch. Power supply of $220 \mathrm{~V}$ is supplied through mains and electrolytic capacitors are used to store the charge and supply current by capacitor discharge method to the heater. Capacitors are connected to switches such that they get charged when the power supply is switched on. Better switch can be replaced in future for changing over the position [9]. 
Recent Advances in Communication, Electronics \& Electrical Engineering

Microcontroller is also used to get the feedback of the switch status at particular instant of time. Depending on the feedback the operation of switches takes place automatically.

Hence by this method a very high current can be supplied to the sample specimen inside the cylinder which is heated at a very high temperature and high pressure. The changes that happen are monitored and recorded. Different parameters are observed and the necessary calculations are performed by the supercomputer.

The computer (PC) is connected to the supercomputer so that the information is sent to the supercomputer from PC where in the supercomputer performs the necessary calculations and computations very quickly and sends back the calculated data to the computer (PC). Hence the result can be obtained very quickly.

Supercomputer acts as the server and the computer acts as the client.

Fig 3 shows the exchange of data between microcontroller and computer, computer and supercomputer. This helps in easier and faster computations.

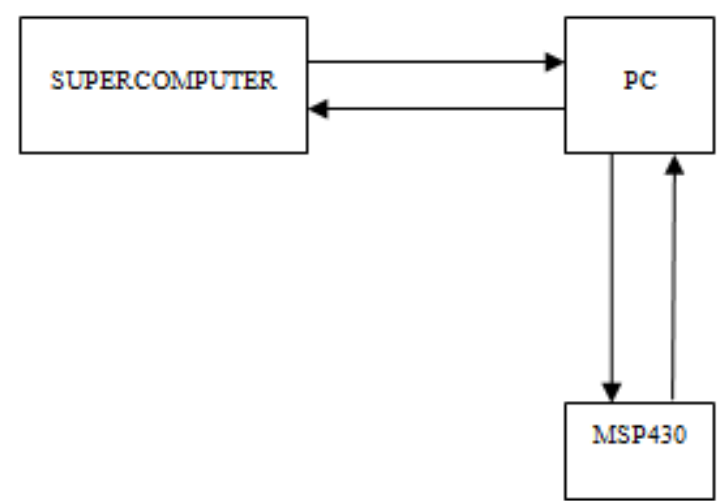

Figure 3: Communication between Supercomputer, computer and microcontroller.

Fig 4 shows the block diagram of high pressure differential thermal analysis system. In differential thermal analysis system, temperature difference between the sample specimen and thermally inert reference substance is continuously monitored as a function of temperature. Phase transformations in materials occurring at elevated conditions of pressure and temperature are investigated by a high pressure DTA. Automated piston cylinder based DTA system is developed to study the phase transformations occurring in materials at high pressure.

As given in Fig 4 the sample specimen is heated by an internal heater [1]. Voltage across the filament is limited to avoid the melting of the filament. Hence higher heating rates are achieved by capacitor discharge method. The millisecond resolution pulse heating system for specific heat measurements consists of an electric pulse power circuit, associated measuring and control circuit, various components and instruments. 
Recent Advances in Communication, Electronics \& Electrical Engineering

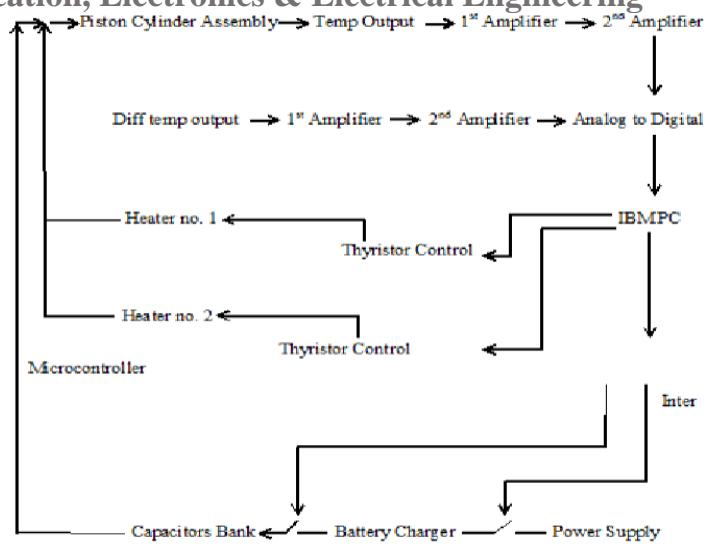

Figure 4: Block Diagram of the High Pressure DTA System

Capacitor discharge heating is an extra amount of heat in the heating element where the regular power supply for the heater is suspended for a while and the capacitor is allowed to discharge during this time. This is performed by the computer with the help of the microcontroller MSP430. The current injection from the discharge method is indicated by the written software program. This happens by the initiation of the interrupt by the computer. From this program in the microcontroller initiates when the interrupt pin of the microcontroller becomes high. Hence computer (PC) can command the microcontroller to give the pulse of heating whenever required.

\section{Experimentation}

The sample is placed inside the cylinder in the pyrophyllite. Sample is directly connected to the current supplier and placed inside the pyrophyllite cell to avoid shorting. Thicker copper plates are used as connectors to the heater. Ceramic beads are used to insulate the connecting wires from the cylinder.

Five switches that are operated by the microcontroller allow the heavy charge of capacitors to pass to the sample specimen through the heating element. Higher heating rates can be reached by varying the capacitance value and this can be achieved by capacitor banks. Capacitor bank has high value of capacitance thus discharging a high surge of current within a short interval of time.

\section{Results}

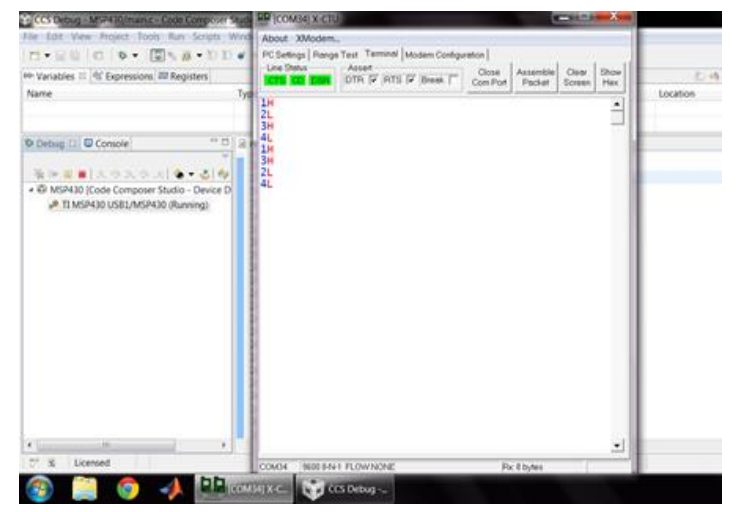

Figure 5: Display of Status of SSR switches. 
Recent Advances in Communication, Electronics \& Electrical Engineering

Fig 5 shows the result for the position of switch control.

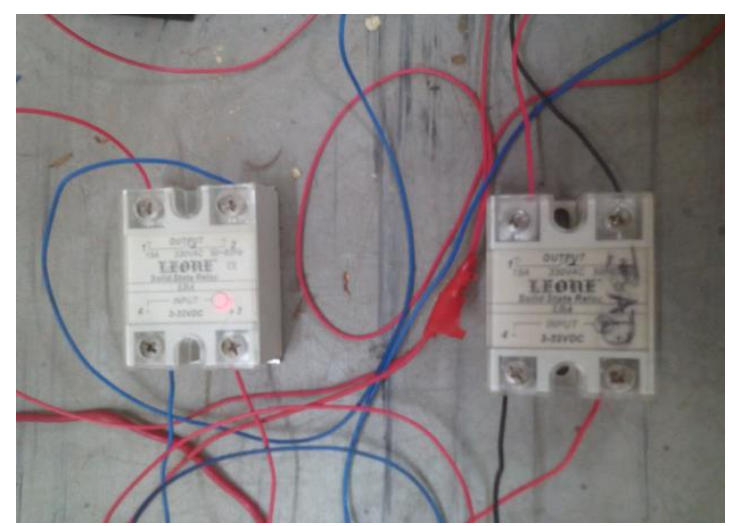

Figure 6: When SSR1 is ON and SSR2 is OFF.

Figure 6 shows the operation of SSR1 alone whereas SSR2 is in OFF state.

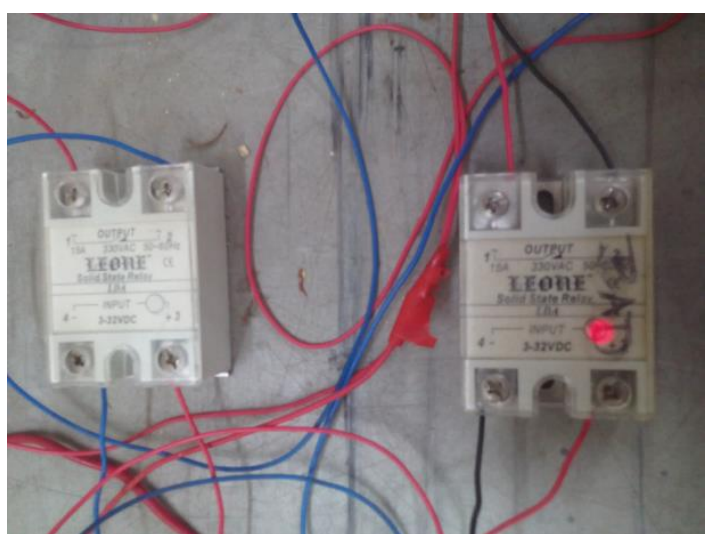

Figure 7: When SSR1 is OFF and SSR2 is ON.

Figure7 shows the operation SSR2 alone whereas SSR1 is in OFF state.

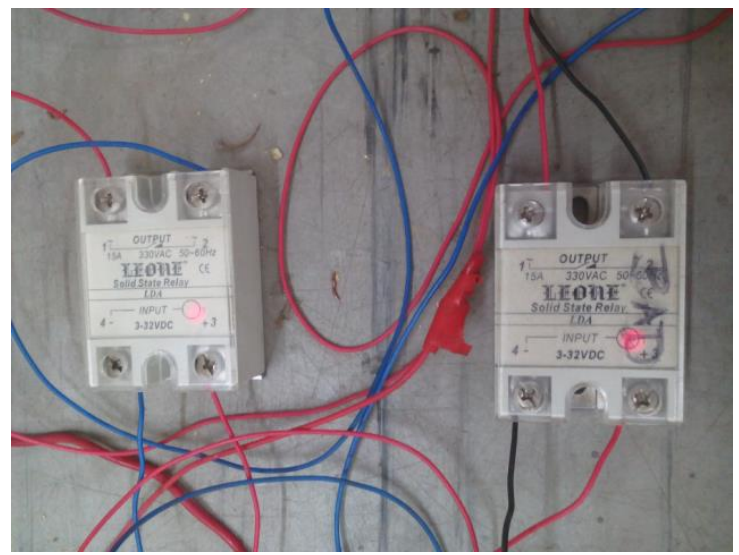

Figure 8: When SSR1 and SSR2 is ON.

Fig 8 shows that both SSR1 and SSR2 are in ON state. 


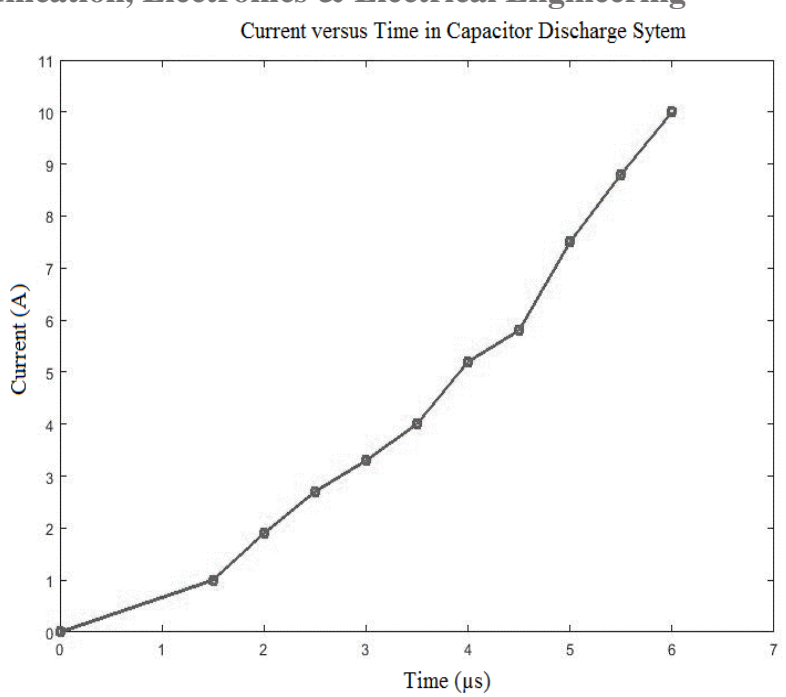

Figure 9: Plot of Time vs. Current

Fig 9 shows the variation of current with respect to time in the capacitor discharge system.

\section{Applications}

Solid state relay switching is useful in: Heating Control, Lighting Control, Motion Control, High-speed switching operation, High-frequency switching operation, Telecommunication and microprocessor control industries, Data communication, TTL or CMOS applications.

Microcontroller MSP430 has been used for SCADA applications [2], composting process [3], for monitoring fuel and tracking the vehicles [4]. From the present work, the application is more useful in rocket engines and supersonic planes.

\section{Conclusion}

Solid state relay switches are controlled using MSP430 to supply the current. PC received the feedback about the status of the particular SSR switch. The particular SSR can then be switched on or off by giving the suitable input through the PC. Contactors connected to the corresponding SSR are energized when the particular SSR is switched on and supplies the current to the sample. Suitable operation of SSR controls the current flow. Rate of current to the sample specimen methane hydrate can be increased by connecting several SSRs in parallel. Instantaneous current is supplied through number of capacitors connected in parallel using Capacitor Discharge method. Necessary current is thus supplied to the sample methane hydrate by the suitable operation of SSRs and through the capacitors. It is observed that the current increased to $10 \mathrm{~A}$ in $6 \mu \mathrm{s}$ by just using four capacitors and the temperature raised up to $0.3^{\circ} \mathrm{C}$ for an individual capacitor. An embedded system circuit has been implemented to ensure that the capacitor banks have a safe and quick charging and discharging process, for the application in the Capacitor Discharge Heating circuit. Remote Procedural Call was used to interface the local PC to the Supercomputer, for fast and reliable analysis of the collected data from the methane hydrate sample. 
Recent Advances in Communication, Electronics \& Electrical Engineering

\section{References}

[1] T K Mondal, "Resistive self-heating of a specimen by a high current pulse from a capacitor discharge system”, Pg. No’s 71-77, Indian Institute of Science.

[2] Nabil Litayem, Manjur Kolhar, Imene Mhadhbi, Saied M. Abd El-atty, Slim Ben Saoud, "Hashing Based Authentication for Ultra-Low Cost Low Power SCADA Application Using MSP430 Microcontroller”, ISSN 2250-2459, Volume 3, Issue 12, December 2013, Pg. No’s 500506.

[3] Prof. Rahane S.B., "An Ultra-Low Powered MSP430 Microcontroller Based Control System for a Composting Process", 2010 International Journal of Computer Applications (0975 -8887), Volume 1 - No. 15, Pg. No's 58-60.

[4] Sachin S. Aher, Kokate R. D., "Fuel Monitoring and Vehicle Tracking", International Journal of Engineering and Innovative Technology (IJEIT), ISSN: 2277-3754, Volume 1, Issue 3, March 2012, Pg. No's 166-169.

[5] Jeetender Singh Chauhan, Sunil Semwal, "Microcontroller Based Speed Control of DC Geared Motor through RS-232 Interface with PC", International Journal of Engineering Research and Applications (IJERA), Dehradun, ISSN: 2248-9622, Vol. 3, Issue 1, January -February 2013, Pg. No's 778-783.

[6] Sonali Mayuresh Akolkar, Bansidhar Eknath Kushare, "A new to improve AC coil contactor switching in presence of voltage sags", Journal of Electrical and Electronic Engineering, Vol. 1, No. 4, 2013, Pg. No's 79-84. doi: 10.11648/j.jeee.20130104.13

[7] Ared Cezairliyan, "A Millisecond-Resolution Pulse Heating System for Specific-Heat Measurements at High Temperatures", Pg. No’s 483-517.

[8] T.Matsumoto, A.Cezairliyan, and D.Basak, "Hemispherical Total Emissivity of Niobium, Molybdenum, and Tungsten at High Electronic Engineering, Vol. 1, No. 4, 2013, Pg. No's 79-84. doi: $10.11648 /$ j.jeee.20130104.13

[9] Ared Cezairliyan, "A Millisecond-Resolution Pulse Heating System for Specific-Heat Measurements at High Temperatures", Pg. No's 483-517.

[10] T.Matsumoto, A.Cezairliyan, and D.Basak, "Hemispherical Total Emissivity of Niobium, Molybdenum, and Tungsten at High Temperatures Using a Combined Transient and Brief Steady-State Technique", International Journal of thermophysics, Vol.20, No.3, 1999, Pg. No's 943-952.

[11] Nwafor Chukwubuikem M., Mbonu Ekene S., Uzedhe Godwin, "A Cost Effective Approach to implementing change over System", Vol. 2, No. 2, March 2012, ISSN-L: 2223-9553, ISSN: 2223-9944, Pg. No's 62-72.

[12] R. Both, C.-I. Muresan, E.-H. Dulf, F.-V. Dulf, "Microcontroller implementation of Digital Fractional Order Controllers for Time Delay Processes", 2013 21st Mediterranean Conference on Control \& Automation (MED) Platanias-Chania, Crete, Greece, June 25-28, 2013, Pg. No's 14451450.

[13] $\mathrm{Lu} \mathrm{Xu}$, Quan Shi, Changzhu chen, Ling sun, Haoran shao, "Design and Implementation of Embedded Web Computer Remote Control System based on MSP430", 2013 Fourth International Conference on Digital Manufacturing \& Automation, Pg. No's 423-427.

[14] Yuang-Shung Lee, Hsin-Wei Huang, Tzu-Han Chou, "Bidirectional DC-DC Converter with Multiple Switched-Capacitor Cells”, 2014 International Power Electronics Conference, Pg. No’s 421-428.

[15] Masiar Babazadeh, Cesare Pautasso, "The Stream Software Connector Design Space: Frameworks and Languages for Distributed Stream Processing”, 2014 IEEE/IFIP Conference on Software Architecture, Pg. No's 1-10.

[16] Shwetabh Srivastava, Pranay Kumar Srivastava, "Performance Analysis of Sun RPC", Engineering and R\&D Services, HCL Technologies Ltd., Noida, India. 
Recent Advances in Communication, Electronics \& Electrical Engineering

[17] Pelle Jakovits, Satish Narayana Srirama, "Evaluating Map Reduce Frameworks for Iterative Scientific Computing Applications", 2014 IEEE, Pg. No's 226-233.

[18] Serkan BIYIK, Murat A YDIN, "A New Life-Test Equipment Designed for Medium-Duty Electromagnetic Contactors", 2014 15th International Conference on Thermal, Mechanical and Multi-Physics Simulation and Experiments in Microelectronics and Microsystems, EuroSimE 2014, Pg. No's 1-7.

\footnotetext{
*Corresponding author.

E-mail address: tk.smitha@gmail.com
} 